\title{
CORRECTION OF FINITE DIFFERENCE EIGENVALUES OF PERIODIC STURM-LIOUVILLE PROBLEMS
}

\author{
ALAN L. ANDREW ${ }^{1}$
}

(Received 7 March 1988; revised 12 July 1988)

\begin{abstract}
Computation of eigenvalues of regular Sturm-Liouville problems with periodic or semiperiodic boundary conditions is considered. A simple asymptotic correction technique of Paine, de Hoog and Anderssen is shown to reduce the error in the centred finite difference estimate of the $k$ th eigenvalue obtained with uniform step length $h$ from $O\left(k^{4} h^{2}\right)$ to $O\left(k h^{2}\right)$. Possible extensions of the results are suggested and the relative advantages of the method are discussed.
\end{abstract}

\section{Introduction}

Considerable recent progress $[1,2,4-9,11,13]$ on the efficient computation of higher Sturm-Liouville eigenvalues (which are much more difficult to compute than the lower eigenvalues) has followed from an idea of Paine, de Hoog and Anderssen [12]. They considered computation of the eigenvalues $\lambda_{1}<\lambda_{2}<\ldots$ of the regular Sturm-Liouville problem

$$
\begin{gathered}
-y^{\prime \prime}+q y=\lambda y \\
y(0)=y(\pi)=0
\end{gathered}
$$

by the centred finite difference method with uniform mesh, and showed that, when $q$ is constant, the error in $\lambda_{k}$ (which in that case is known in closed form) has the same asymptotic form as $k \rightarrow \infty$ as the error for general $q$. They showed that the accuracy of the estimates obtained for high eigenvalues could be dramatically improved at negligible extra cost by using the known error for $q=0$ to correct the estimates obtained for general $q$. Their numerical results

\footnotetext{
${ }^{1}$ Mathematics Department, La Trobe University, Bundoora, Victoria 3083, Australia.

(C) Copyright Australian Mathematical Society 1989, Serial-fee code 0334-2700/89
} 
indicate that this correction generally improves the accuracy of all computed eigenvalues, not just the higher ones.

Anderssen and de $\operatorname{Hoog}[1,2]$ extended the analysis of [12] to problems with the general separated boundary conditions

$$
\sigma_{1} y^{\prime}(0)+\sigma_{2} y(0)=\sigma_{3} y^{\prime}(\pi)+\sigma_{4} y(\pi)=0 .
$$

One difficulty with (1), (3) is that closed form solutions for the error when $q$ is constant are generally no longer available, though an efficient means of computing that error is suggested in [2]. Three important exceptions not emphasised in $[1,2]$ are

$$
\begin{gathered}
y^{\prime}(0)=y^{\prime}(\pi)=0 \\
y(0)=y^{\prime}(\pi)=0
\end{gathered}
$$

and

$$
y^{\prime}(0)=y(\pi)=0 .
$$

When $n$ (equal) subintervals are used, corrected eigenvalues are obtained by adding to the centred finite difference estimate of $\lambda_{k},(k-1)^{2}-4 \sin ^{2}[(k-$ 1) $h / 2)] / h^{2}$ in the case of (4) and $\left(k-\frac{1}{2}\right)^{2}-4 \sin ^{2}[(2 k-1) h / 4] / h^{2}$ in the case of (5) or (6), where $h=\pi / n$. This correction has been used successfully in a numerical investigation of acoustic propagation problems in stratified oceans [13]. This involves a problem of the form (1), (5).

There are also several examples involving nonseparated boundary conditions in which closed form solutions are available, but not all are suitable candidates for this "asymptotic" correction technique. For example, with the (non-selfadjoint) boundary conditions

$$
y(0)=-y(\pi), \quad y^{\prime}(0)=y^{\prime}(\pi)
$$

or

$$
y(0)=y(\pi), \quad y^{\prime}(0)=-y^{\prime}(\pi),
$$

all real numbers are eigenvalues of (1) when $q$ is constant. For all real $c, y$ is then an eigenfunction with (7) if $y(x)=\sinh [c(x-\pi / 2)], y(x)=\sin [c(x-\pi / 2)]$ or $y(x)=x-\pi / 2$, while the derivatives of these functions are eigenfunctions with (8).

Important nonseparated boundary conditions for which the correction technique can be used successfully are the periodic boundary conditions

$$
y(0)=y(\pi), \quad y^{\prime}(0)=y^{\prime}(\pi)
$$

and the semiperiodic boundary conditions

$$
y(0)=-y(\pi), \quad y^{\prime}(0)=-y^{\prime}(\pi) .
$$

Periodic boundary conditions arise in the study of planetary orbits and other periodic physical phenomena (including some models for crystal structure) and of 
course when polar co-ordinates are used in partial differential equations solved by the method of separation of variables. Problems with these boundary conditions in applications [10] usually satisfy

$$
q(0)=q(\pi) .
$$

Use of the correction technique in conjunction with the finite element method was studied in [8] in the special case (2) and later in [5] this analysis was extended to the boundary conditions (4), (5), (6), (9) and (10). However, no analysis has yet been done of the correction technique with finite differences when the boundary conditions are not separated. This paper gives this analysis for the important nonseparated boundary conditions (9) and (10) and compares the advantages of the finite difference and finite element approaches. Whereas the results in [1], [2] and [12] required $k h$ to be "sufficiently small", our proof, which uses ideas from [5] and [7] and is readily adapted to cover the boundary conditions (2), (4), (5) and (6), is valid for all computed eigenvalues.

\section{Analysis of the correction}

The centred finite difference method with mesh length $h$ approximates the first $n$ eigenvalues, $\lambda_{1}^{(4)}, \ldots, \lambda_{n}^{(4)}$, of $(1),(9)$ by the eigenvalues, $\Lambda_{1}^{(4, n)} \leq \cdots \leq \Lambda_{n}^{(4, n)}$ of the matrix $-\mathbf{A}^{(4)}+\mathbf{Q}$, and the first $n$ eigenvalues, $\lambda_{1}^{(5)}, \ldots, \lambda_{n}^{(5)}$, of (1), (10) by the eigenvalues $\Lambda_{1}^{(5, n)} \leq \cdots \leq \Lambda_{n}^{(5, n)}$ of $-\mathbf{A}^{(5)}+\mathbf{Q}$, where

$$
\mathbf{Q}=\operatorname{diag}\left[q\left(x_{1}\right), \ldots, q\left(x_{n}\right)\right], \quad x_{i}=i h(i=1, \ldots, n)
$$

and the matrices $\mathbf{A}^{(4)}, \mathbf{A}^{(5)}$ are as defined in [5]. That is, the element in the $i$ th row and $j$ th column of $\mathbf{A}^{(4)}$ is $-2 / h^{2}$ (if $i=j$ ), $1 / h_{2}$ (if $|i-j|=1$ or $n-1$ ) and 0 (if $1<|i-j|<n-1$ ), while $\mathbf{A}^{(5)}$ is obtained from $\mathbf{A}^{(4)}$ by changing the sign of the elements in the $(1, n)$ and $(n, 1)$ positions.

When $q=0$ then, as noted in [5],

$$
\lambda_{k}^{(4)}=K_{1}^{2} \quad \text { and } \quad \lambda_{k}^{(5)}=K_{2}^{2}
$$

where

$$
K_{1}=2[k / 2] \text { and } K_{2}=2[(k+1) / 2]-1
$$

and, in (13), $[x]$ denotes the greatest integer not exceeding $x$. Moreover it is readily checked that

$$
-\mathbf{A}^{(p)} \mathbf{s}_{k}^{(p)}=\mu_{k}^{(p, n)} \mathbf{s}_{k}^{(p)}, \quad k=1, \ldots, n, \quad p=4,5
$$

where

$$
\begin{aligned}
& \mu_{k}^{(4, n)}=4 \sin ^{2}\left(K_{1} h / 2\right) / h^{2}, \\
& \mu_{k}^{(5, n)}=4 \sin ^{2}\left(K_{2} h / 2\right) / h^{2},
\end{aligned}
$$


all components of $\mathbf{s}_{1}^{(4)}$ are equal and, for $m>0$, the $i$ th components of $s_{2 m}^{(4)}$, $\mathbf{s}_{2 m+1}^{(4)}, \mathbf{s}_{2 m-1}^{(5)}$ and $\mathbf{s}_{2 m}^{(5)}$ are $\cos (2 m i h), \sin (2 m i h), \cos [(2 m-1) i h]$ and $\sin [(2 m-$ 1) $i h$ ] respectively. Hence the finite difference eigenvectors again give the values of the exact eigenfunctions at the mesh points when $q$ is constant.

Theorem 1 below shows that, at least for large $k$, the corrected estimates

$$
\tilde{\Lambda}_{k}^{(4, n)}=\Lambda_{k}^{(4, n)}+K_{1}^{2}-\mu_{k}^{(4, n)}
$$

and

$$
\widetilde{\Lambda}_{k}^{(5, n)}=\Lambda_{k}^{(5, n)}+K_{2}^{2}-\mu_{k}^{(5, n)}
$$

are much better estimates than $\Lambda_{k}^{(4, n)}$ and $\Lambda_{k}^{(5, n)}$ of the true eigenvalues $\lambda_{k}^{(4)}$ and $\lambda_{k}^{(5)}$.

The restriction $\sin \left(K_{i} h\right) \neq 0$ is also required for the corresponding results for $(4),(9)$ and (10) in [5] and [6] to be meaningful. This is not a severe restriction as, apart from the case $k=1$ with (1), (9) (when the correction produces no change), $\sin \left(K_{i} h\right)$ in Theorem 1 can be zero only when $k=n$ and then only for (1), (9) when $n$ is even and for (1), (10) when $n$ is odd. Moreover, the analysis here and in [5] is readily extended to the case $\sin \left(K_{i} h\right)=0$ by making a minor extension to Lemma 5 of [7] and Lemma 2.2 of [12]. If $k=n+1$ in Lemma 5 of [7], the bound in that lemma becomes $(n+1)\|\theta\|_{\infty}$. When $\beta=p \pi$ ( $p$ an integer) in Lemma 2.2 of [12], all terms in the sum there have value one. Using these extensions of Lemma 5 of [7] and Lemma 2.2 of [12] to extend cited results from [5], [7], [8] and [12], shows that $\widetilde{\Lambda}_{n}^{(p, n)}-\lambda_{n}=O(1)$ for $p=4$ and 5 even when $\sin \left(K_{i} h\right)=0$. Since our methods are most useful when $k<n$ (see Section 3), details of the analysis in the exceptional case $\sin \left(K_{2} h\right)=0$ (and the corresponding cases in [5]) are left to the reader.

THEOREM 1. For each $q \in C^{2}[0, \pi]$ satisfying (11), there exist constants $c_{1}, c_{2}$, depending only on $q$, such that, for all $n \in N$,

$$
\left|\tilde{\Lambda}_{k}^{(4, n)}-\lambda_{k}^{(4)}\right| \leq c_{1} K_{1}^{2} h^{3} / \sin \left(K_{1} h\right), \quad k=2, \ldots, n
$$

and

$$
\left|\tilde{\Lambda}_{k}^{(5, n)}-\lambda_{k}^{(5)}\right| \leq c_{2} K_{2}^{2} h^{3} / \sin \left(K_{2} h\right), \quad k=1, \ldots, n,
$$

provided the denominator on the right hand side is non zero.

PROOF. We outline the proof for (9) only. The proof for (10) is exactly similar. For notational convenience, the subscript $k$ and the superscripts $(4)$ and $(4, n)$ are suppressed throughout the proof. For any function $g:[0, \pi] \rightarrow R$, we write $g=$ $\left(g\left(x_{1}\right), \ldots, g\left(x_{n}\right)\right)^{\top}, \mathbf{g}^{\prime \prime}=\left(g^{\prime \prime}\left(x_{1}\right), \ldots, g^{\prime \prime}\left(x_{n}\right)\right)^{\top}$ etc. Without loss of generality we assume

$$
\int_{0}^{\pi} q(x) d x=0 .
$$


This implies $[5,10]$ that

$$
\lambda=K_{1}^{2}+O\left(K_{1}^{-2}\right) .
$$

Our first step is to establish the existence of real numbers $\theta$ and $\psi$ such that, when $y$ and the solution $\mathbf{u}$ of the difference equations below are suitably normalised,

$$
y=s_{\theta}+e
$$

and

$$
\mathbf{u}=\mathbf{s}_{\psi}+\varepsilon
$$

where

$$
s_{\theta}(x)=\sin \left(K_{1} x+\theta\right), s_{\psi}(x)=\sin \left(K_{1} x+\psi\right)
$$

and $e$ and $\varepsilon$ are "small" for large $k$. In fact (21) was proved in the proof of Theorem 2 of [5], where it was shown that

$$
e(x)=K_{1}^{-1} \int_{0}^{x} f(t) \sin \left[K_{1}(x-t)\right] d t
$$

where $f=\left(K_{1}^{2}-\lambda+q\right) y$, and it was also shown that, if $q \in C^{2}[0, \pi]$, then

$$
e^{\prime \prime}+K_{1}^{2} e=f
$$

and

$$
\mathbf{s}_{\psi}^{\top} \mathbf{f}=-h \int_{0}^{\pi} P_{2}(x / h)\left(s_{\psi} f\right)^{\prime \prime}(x) d x+O(h)=O\left(K_{1}^{2} h^{2} / \sin \left(K_{1} h\right)\right)
$$

where the piecewise polynomial $P_{2}$ is defined in [5].

The difference equations

$$
\left(2 u_{i}-u_{i+1}-u_{i-1}\right) / h^{2}+q\left(x_{i}\right) u_{i}=\Lambda u_{i}, \quad i=0, \ldots, n
$$

and boundary conditions $u_{0}=u_{n}, u_{1}-u_{-1}=u_{n+1}-u_{n-1}$, from which the matrix $-\mathbf{A}^{(4)}+\mathbf{Q}$ is constructed, imply that $u_{1}=u_{n+1}$. Hence the arguments used to prove Theorem 2.1 and Corollary 2.1 of [12] (and also Lemma 1 of [7]) show that (22) is also true with

$$
\varepsilon_{i}=\sum_{j=1}^{i-1} \sin \left[K_{1} h(i-j)\right] h^{2}\left(q_{j}+\mu-\lambda\right) u_{j} / \sin \left(K_{1} h\right)
$$

and hence

$$
\|\varepsilon\|_{\infty} \leq 2 h \pi\|q\|_{\infty}\|\mathrm{u}\|_{\infty} / \sin \left(K_{1} h\right) .
$$

As in the proof of Theorem 2 of [5] (see also equation (17) of [7]), it follows from (1), (14), (21), (22), (23) and (25) that

$$
\begin{aligned}
(\Lambda-\lambda) \mathbf{u}^{\top} \mathbf{y} & =\left(\mu-K_{1}^{2}\right) \mathbf{u}^{\top} \mathbf{s}_{\theta}+\mathbf{s}_{\psi}^{\top}\left(\mathbf{e}^{\prime \prime}-\mathbf{A e}\right)+\varepsilon^{\top}\left(\mathbf{e}^{\prime \prime}-\mathbf{A e}\right) \\
& =\left(\mu-K_{1}^{2}\right) \mathbf{u}^{\top} \mathbf{y}+\mathbf{s}_{\psi}^{\top} \mathbf{f}+\varepsilon^{\top}(\mathbf{f}-\mathbf{A e}-\mu \mathbf{e}) .
\end{aligned}
$$


Now, since by (21) $e(0)=e(\pi)$ and $e^{\prime}(0)=e^{\prime}(\pi)$, the argument used for the first part of the proof of Lemmas 3 and 4 of [7] (and also Lemma 3 of [8]) shows that

$$
\mathrm{Ae}+\mu \mathrm{e}=\mathrm{E} / K_{1} h^{2}
$$

where, for $j=1, \ldots, n$,

$$
\begin{aligned}
E_{j} & =\int_{x_{j}}^{x_{j+1}} f(t) \sin \left[K_{1}\left(x_{j+1}-t\right)\right] d t+\int_{x_{j}}^{x_{j-1}} f(t) \sin \left[K_{1}\left(x_{j-1}-t\right)\right] d t \\
& =\left(h^{2} \mu / K_{1}\right) f_{j}+O\left(K_{1} h^{4}\left\|f^{\prime \prime}\right\|_{\infty}\right)
\end{aligned}
$$

(In the definition of $E_{n}, f$ is assumed to have period $\pi$.)

Hence, since $\left\|f^{\prime \prime}\right\|_{\infty}=O\left(K_{1}^{2}\right)$ and, by (15), $\mu=K_{1}^{2}+O\left(K_{1}^{4} h^{2}\right)$, it follows from (28) and Cauchy's inequality that

$$
\left|\varepsilon^{\top}(\mathbf{f}-\mathbf{A e}-\mu \mathbf{e})\right|=O\left(K_{1}^{2} h^{2} / \sin \left(K_{1} h\right)\right) .
$$

Hence by (17), (26) and (29) $|\tilde{\Lambda}-\lambda|\left|\mathbf{u}^{\top} \mathbf{y}\right|=O\left(K_{1}^{2} h^{2} / \sin \left(K_{1} h\right)\right)$ and the result follows by the concluding arguments used in the proof of Theorem 1 of [7] (also used in [8] and [5]). The remarks following the proof of Theorem 1 of [5] also apply.

\section{Numerical results and discussion}

In order to facilitate comparison with the results of [5], the same $q$ was chosen for numerical computation, namely

$$
q(x)=10 \cos (2 x) .
$$

Since this $q$ is even about $\pi / 2$, the eigenfunctions of (1), (9), (32) and those of (1), (10), (32) and (1), (2), (32) and (1), (4), (32) are either even or odd about $\pi / 2$ (cf. [3]). From this it can be deduced that, with (32), in contrast to most $q$ including those satisfying (11), the even eigenvalues $\lambda_{2 m}$ and corresponding eigenfunctions of (1), (9) are precisely the even ones of (1), (2) and the odd eigenvalues and eigenfunctions of (1), (9) are the odd ones of (1), (4), while the eigenvalues and eigenfunctions of (1), (10) are precisely the remaining ones of (1), (2) and (1), (4). This special property of (32) was used to compute some "exact" eigenvalues by the method of [7] for comparison with those computed by the method described here.

For $k=1, \ldots, n$ the uncorrected and corrected estimates $\Lambda_{k}^{(n)}$ and $\widetilde{\Lambda}_{k}^{(n)}$ were computed for (1), (9) and (1), (10) for $n=5,10,20,40$ and 80 and for (1), (9) also for $n=4,8,12$ and 16. Some representative results are shown in Table 1. From left to right the columns of Table 1 show, for various $k$, the exact eigenvalue, the error in the uncorrected finite difference estimate with $n=80$, 
and the errors in the corrected estimates with $n=80,40$ and 20 respectively. The correction changes all computed eigenvalues for (1), (10) and all except $k=1$ (which is consequently omitted from Tables 1 and 2) for (1), (9), and in all the author's calculations this change produced an improvement. Moreover Table 1 shows that, for all $k>4$, the corrected results for (1), (9) with $n=20$ are better than the uncorrected results with $n=80$ which require much more computation and, as predicted by the theory, the improvement produced by the correction increases rapidly with $k$.

TABLE 1. Results for (1), (9), with (32)

\begin{tabular}{|r|r|r|r|r|r|}
\hline$k$ & \multicolumn{1}{|c|}{$\lambda_{k}$} & $\lambda_{k}-\Lambda_{k}^{(80)}$ & $\lambda_{k}-\widetilde{\Lambda}_{k}^{(80)}$ & $\lambda_{k}-\widetilde{\Lambda}_{k}^{(40)}$ & $\lambda_{k}-\widetilde{\Lambda}_{k}^{(20)}$ \\
\hline 2 & 2.09946 & 0.006 & 0.0044 & 0.0175 & 0.0714 \\
3 & 7.44911 & 0.008 & 0.0058 & 0.0235 & 0.0974 \\
4 & 16.64822 & 0.037 & 0.0041 & 0.0169 & 0.0745 \\
5 & 17.09658 & 0.034 & 0.0011 & 0.0048 & 0.0242 \\
6 & 36.35887 & 0.169 & 0.0031 & 0.0134 & 0.0687 \\
7 & 36.36090 & 0.169 & 0.0031 & 0.0131 & 0.0675 \\
8 & 64.19884 & 0.528 & 0.0033 & 0.0145 & 0.0898 \\
10 & 100.12637 & 1.282 & 0.0034 & 0.0158 & 0.1264 \\
12 & 144.08745 & 2.649 & 0.0034 & 0.0175 & 0.1965 \\
14 & 196.06412 & 4.891 & 0.0036 & 0.0197 & 0.3575 \\
16 & 256.04903 & 8.316 & 0.0037 & 0.0225 & 1.1214 \\
18 & 324.03870 & 13.272 & 0.0038 & 0.0263 & 3.3904 \\
20 & 400.03133 & 20.148 & 0.0040 & 0.0313 & -5.7942 \\
30 & 900.01390 & 99.400 & 0.0052 & 0.1244 & - \\
38 & 1444.00866 & 249.850 & 0.0071 & 3.4343 & - \\
40 & 1600.00782 & 303.097 & 0.0078 & -5.7986 & - \\
\hline
\end{tabular}

Theorem 1 and the remarks preceding it show that

$$
\tilde{\Lambda}_{n}^{(4, n)}-\lambda_{n}=O(1)
$$

and numerical results show this estimate to be sharp. Indeed, with (1), (9), (32), $5.79<\widetilde{\Lambda}_{n}^{(4, n)}-\lambda_{n}<5.8$ for $n=16,20,40$ and 80 . Similar results hold, with similar proofs, for (2), (4), (5), (6) and (10), and numerical results show these estimates to be sharp also. Also, with (1), (9), (32), the growth with $k$ of $\left|\lambda_{n}-\widetilde{\Lambda}^{(4, n)}\right|$ for $n / 4<k<3 n / 4$ is not much less than the growth of the bound of Theorem 1. However, although, for $k \leq n / 2$, the bound of Theorem 1 is effectively $O\left(k h^{2}\right)$, numerical results for (1), (9), (32) shows that $\left|\lambda_{m}-\widetilde{\Lambda}_{m}^{(4,4 m)}\right|<$ $\left|\lambda_{2}-\tilde{\Lambda}_{2}^{(4,4 m)}\right|$ for $m=5,10$ and 20 and suggest that

$$
\lambda_{m}-\widetilde{\Lambda}_{m}^{(4,2 m)}=O\left(m^{-2}\right),
$$

showing that, for $k<n$, the bound of Theorem 1 is not sharp.

Sharper bounds could perhaps be obtained by adapting the (lengthier) proof given for (3) in [1] and summarised in [2]. (See also the comments in [5].) An 
alternative approach is to sharpen the estimates obtained here. The argument of Lemma 6 of [7] shows that, when $q \in C^{4}[0, \pi]$ (as in the case for (32) and all $q$ used in $[1,2,4-8,12]),(26)$ can be strengthened to $\mathrm{s}_{\psi}^{\top} \mathrm{f}=O\left(K_{1}^{4} h^{4} / \sin \left(K_{1} h\right)\right)+O(h)$. For sufficiently small $K_{1} h$, the first term will be dominated by the second and, for sufficiently smooth $q$, may perhaps be reduced further by taking more terms in the Euler-Maclaurin expansion in the proof in [7]. Since also (27) and (30) contain more information than (31), it should be possible to adapt the proof of Theorem 1 to obtain sharper results for "small" $k$. Sharpening the results of Theorem 1 (and of course the corresponding results of [2], [5], [7], [8] and [12]) may be of more than theoretical interest as it may increas the range of $k h$ for which extrapolation may be successfully applied to the corrected estimates, $\widetilde{\Lambda}_{k}^{(n)}$. As with the method of [8], Table 1 shows that, for moderately small $k h$, the accuracy of the corrected estimates may be improved substantially by simple $h^{2}$ extrapolation, but that for larger values of $k h$ a more appropriate extrapolation formula is required.

Comparison of Table 1 with Table 2 of [5] shows that, at least for (32), the corrected second order finite difference estimates studied here are generally less accurate than the corrected second order finite element estimates of [5], especially when $n$ is close to or slightly less than $2 k$. Indeed, with (1), (9), (32), it is easy to show that, when $m=1$,

$$
\widetilde{\Lambda}_{2 m}^{(4,4 m)}=\widetilde{\Lambda}_{2 m+1}^{(4,4 m)}=4 m^{2},
$$

and numerical results suggest that (35) is true for all positive integers $m$. (Note that, by (20), (35) implies (34).) Since (32) implies (19), the corrected finite difference estimates in (35) are exactly the same as the simple asymptotic estimate of (20) and much worse than the corrected finite element estimates of [5]. (To keep this result in perspective, it should be noted that, for $n>2 k$, the corrected finite difference estimate was always better than the asymptotic estimate (20), with the superiority of the methods of this paper increasing rapidly as $k h$ decreases, as predicted by (20) and Theorem 1.) There is also some numerical evidence [9] that the correction technique can be extended to higher order finite element methods and this is likely to lead to a great improvement in accuracy, just as the method of [7] is much more accurate than that of [12]. (See the remarks in [8].)

However there are two advantages of the finite difference approach of this paper over the finite element approach of [5]. First, the method of [5] requires the solution of an eigenvalue problem of the form $\mathbf{A x}=\lambda \mathbf{B x}$ with $\mathbf{B} \neq \mathbf{I}$, whereas with the method described here $\mathbf{B}=\mathbf{I}$. The most easily used software packages from sources such as EISPACK for solving $\mathbf{A x}=\lambda \mathbf{B x}$ with $\mathbf{B} \neq \mathbf{I}$ do not take advantage of the special periodic tridiagonal structure of $\mathbf{A}$ and $\mathbf{B}$ for this problem, possibly because of the philosophy adopted in [14] of including 
only routines which were in some sense optimal. Although the author found no difficulty with the method described in [5] for solving the matrix problem, it has not had the extensive testing of the EISPACK routines. Secondly, the finite element approach requires the numerical evaluation of a number of integrals not required for the finite difference approach. A user wishing to compute, say, the first 20 or 30 eigenvalues with uniform accuracy significantly greater than that obtainable from the simple asymptotic formula (even for the greatest of these), but who does not require the still greater accuracy which can be obtained by the corrected finite element method, and who wishes to use packaged software, is likely to find the method of this paper easier to implement than that of [5].

TABLE 2. Results for (1), (9), with (36)

\begin{tabular}{|r|r|r|c|r|}
\hline \multicolumn{1}{|c|}{$k$} & \multicolumn{1}{|c|}{$\lambda_{k}$} & $\lambda_{k}-\Lambda_{k}^{(40)}$ & $\lambda_{k}-\widetilde{\Lambda}_{k}^{(40)}$ & \multicolumn{1}{c|}{$\lambda_{k}-\widetilde{\Lambda}_{k}^{(20)}$} \\
\hline 2 & 6.5005 & 0.0117 & 0.0035 & 0.0144 \\
3 & 7.0151 & 0.0084 & 0.0002 & 0.0006 \\
4 & 18.5848 & 0.1347 & 0.0036 & 0.0152 \\
5 & 18.6655 & 0.1318 & 0.0006 & 0.0024 \\
6 & 38.5816 & 0.6651 & 0.0038 & 0.0173 \\
7 & 38.6215 & 0.6619 & 0.0006 & 0.0022 \\
8 & 66.5821 & 2.0820 & 0.0040 & 0.0200 \\
9 & 66.6054 & 2.0786 & 0.0006 & 0.0017 \\
10 & 102.5825 & 5.0401 & 0.0042 & 0.0246 \\
11 & 102.5977 & 5.0365 & 0.0006 & 0.0010 \\
12 & 146.5829 & 10.3529 & 0.0044 & 0.0332 \\
13 & 146.5935 & 10.3491 & 0.0005 & -0.0005 \\
14 & 198.5831 & 18.9732 & 0.0047 & 0.0527 \\
15 & 198.5910 & 18.9690 & 0.0005 & -0.0040 \\
16 & 258.5833 & 31.9691 & 0.0050 & 0.1155 \\
17 & 258.5893 & 31.9645 & 0.0004 & -0.0173 \\
18 & 326.5834 & 50.4981 & 0.0055 & 0.5571 \\
19 & 326.5882 & 50.4930 & 0.0004 & -0.2362 \\
20 & 402.5835 & 75.7783 & 0.0061 & -0.5197 \\
\hline
\end{tabular}

Following a suggestion of a referee that some numerical results for an example with nonsymmetric $q$ be added, some calculations were made with

$$
q(x)=x^{2}(\pi-x) \text {. }
$$

The simple method of calculating "exact" solutions used for (32) is not available for (36), but the "exact" solutions of Table 2 were obtained by extrapolating the corrected results obtained by the method of this paper with $n=100$ and $n=125$. Comparison with solutions obtained by extrapolation of corrected results for $n=80$ and $n=100$ suggests that all figures shown in Table 2 are correct.

In general the results obtained for (1), (9), (36) were similar to those obtained for (1), (9), (32) and in particular they satisfied (33). There was however one notable difference which is probably due to the fact that the pairs of eigenvalues 
of (1), (9), (32) coalesce much more rapidly than those of (1), (9), (36) as $k$ increases. Although in both cases the corrected eigenvalue estimates for (1), (9) were generally more accurate for odd $k$ than for even $k$, the difference for midrange $k$ was negligible with (32). With (1), (9), (36) the difference was quite significant, and, whereas for even $k$ the error in the corrected estimates increased monotonically with $k$, for odd $k$ it actually decreased as $k$ increased, for midrange $k$. This is demonstrated by Table 2 which, for $k=2$ to 20 , gives, in successive columns, the exact eigenvalues, the error in the uncorrected estimates for $n=40$ and the error in the corrected estimates for $n=40$ and $n=20$ respectively for (1), (9), (36). It may be relevant that the odd eigenvalues of (1), (9), (36) are much closer than the even eigenvalues to eigenvalues of (1), (2), (36).

\section{References}

[1] R. S. Anderssen and F. R. de Hoog, "On the correction of finite difference eigenvalue approximations for Sturm-Liouville problems with general boundary conditions", Tech. Rep. CMA-R05-82, Centre for Math. Analysis, Australian National Univ., 1982.

[2] R. S. Anderssen and F. R. de Hoog, "On the correction of finite difference eigenvalue approximations for Sturm-Liouville problems with general boundary conditions", BIT 24 (1984) 401-412.

[3] A. L. Andrew, "Eigenvectors of certain matrices", Linear Algebra Appl. 7 (1973) 151-162.

[4] A. L. Andrew, "Asymptotic correction of finite difference eigenvalues", in Computational techniques and applications: CTAC-85 (eds. J. Noye and R. May.) (North-Holland, Amsterdam, 1986) 333-341.

[5] A. L. Andrew, "Correction of finite element eigenvalues for problems with natural or periodic boundary conditions", BIT 28 (1988) 254-269.

[6] A. L. Andrew, "Some recent developments in finite element eigenvalue computation", in Computational techniques and applications: CTAC-87 (eds. J. Noye and C. Fletcher.) (North-Holland, Amsterdam, 1988) 83-91.

[7] A. L. Andrew and J. W. Paine, "Correction of Numerov's eigenvalue estimates", Numer. Math. 47 (1985) 289-300.

[8] A. L. Andrew and J. W. Paine, "Correction of finite element estimates for Sturm-Liouville eigenvalues", Numer. Math. 50 (1986) 205-215.

[9] G. Doherty, M. J. Hamilton, P. G. Burton and E. I. von Nagy-Felsobuki, "A numerical variational method for calculating accurate vibrational energy separations of small molecules and their ions", Austral. J. Phys. 39 (1986) 749-760.

[10] M. S. P. Eastham, The spectral theory of periodic differential equations (Scottish Academic Press, Edinburgh, 1973).

[11] J. Paine, "A numerical method for the inverse Sturm-Liouville problem", SIAM J. Sci. Stat. Comput. 5 (1984) 149-156.

[12] J. W. Paine, F. R. de Hoog and R. S. Anderssen, "On the correction of finite difference eigenvalue approximations for Sturm-Liouville problems", Computing 26 (1981) 123-139.

[13] M. Porter and E. L. Reiss, "A numerical method for ocean-acoustic normal modes", $J$. Acoust. Soc. Amer. 76 (1984) 244-252.

[14] J. H. Wilkinson and C. Reinsch, Handbook for automatic computation, Vol. II, Linear algebra (Springer, New York, 1971). 